\title{
The Impact of Obesity on Performing Lymphadenectomy and Its Outcome in Ovarian Cancer Patients
}

\author{
Basel Refky1, Mosab Shetiwy ${ }^{1}$, Ahmed Zaki², Mohamed Elmetwally', Hanan Nabil2, \\ Islam Abdou1, Ahmed Abdallah'1, Amr Soliman'3 , Khaled Abdelwahab1, Anas Gamal2, \\ Essam Elshiekh4, Khaled Gaballa ${ }^{*}$ \\ ${ }^{1}$ Oncology Center Mansoura University, Mansoura, Egypt \\ ${ }^{2}$ Mansoura University Hospitals, Mansoura, Egypt \\ ${ }^{3}$ Oldenburg University, Oldenburg, Germany \\ ${ }^{4}$ Tanta Cancer Center, Tanta, Egypt \\ Email: dr.basel@hotmail.com, mosabsaad@mans.edu.eg, dr_a_zaki@mans.edu.eg, mohamedocmu@gmail.com, \\ hanannobi100@yahoo.com, islamabdouu2010@yahoo.com,drabdallah82@gmail.com, amr.soliman@uni-oldenburg.de, \\ Khaled14eg@hotmail.com, Anasgamal@gmail.com, essam.elshikh@gmail.com, ^khgab@mans.edu.eg
}

How to cite this paper: Refky, B., Shetiwy, M., Zaki, A., Elmetwally, M., Nabil, H., Abdou, I., Abdallah, A., Soliman, A., Abdelwahab, K., Gamal, A., Elshiekh, E. and Gaballa, K. (2018) The Impact of Obesity on Performing Lymphadenectomy and Its Outcome in Ovarian Cancer Patients. Journal of Cancer Therapy, 9, 568-575. https://doi.org/10.4236/jct.2018.97048

Received: June 25, 2108

Accepted: July 20, 2018

Published: July 23, 2018

Copyright $\odot 2018$ by authors and Scientific Research Publishing Inc. This work is licensed under the Creative Commons Attribution International License (CC BY 4.0).

http://creativecommons.org/licenses/by/4.0/

\section{Open Access}

\begin{abstract}
Background: This study discusses the effect of obesity on the number of lymph nodes harvested during systematic LND and the LND-related complications in ovarian cancer patients. Methods: This retrospective study enrolled women with ovarian cancer who were consecutively subjected to open surgical resection that included systematic LND (pelvic and para-aortic) in the Oncology Center in Mansoura University (OCMU) during the period between January 2012 and June 2017. Patients were categorized according to the recommendations of World Health Organization by their BMI as non-obese $\left(B M I<30.0 \mathrm{~kg} / \mathrm{m}^{2}\right)$ and obese $\left(B M I \geq 30.0 \mathrm{~kg} / \mathrm{m}^{2}\right)$. Results: Seventy-seven women with ovarian cancer were enrolled in the study according to our inclusion and exclusion criteria. 43 females (55.8\%) were grouped as obese and 34 $(44.2 \%)$ as non-obese. Lymph nodes retrieved in total and in different stations separately (pelvic and para-aortic) were all similar among patients in both groups. LND-related intraoperative complications were observed in 8 patients $(18.6 \%)$ in the obese group and 3 patients in the non-obese group $(8.8 \%)(\mathrm{P}=$ $0.347)$. Hospital stay was the same in the two groups with a median of 4 days (IQR 3 - 5). Postoperative complications occurred in 13 patients $(30.2 \%)$ in the obese group and only in one patient $(3 \%)$ in the non-obese group $(\mathrm{P}=$ 0.004). Conclusion: Obese ovarian cancer patients may safely undergo comprehensive staging involving extensive lymph node dissection in open surgeries without significant increase in the rates of intraoperative complications.
\end{abstract}


Whereas, postoperative complications (wound infection and thromboembolic events) tend to occur at higher rates with obese patients. Trial registration: This study was retrospectively registered and approved at faculty of Medicine Mansoura University, Egypt with IRB approval number R.18.02.46.

\section{Keywords}

Lymphadenectomy, Obesity, Ovarian Cancer

\section{Introduction}

Ovarian cancer staging surgery involves systemic iliac and para-aortic lymphadenectomy for early stages and removal of bulky lymph nodes for advanced stages [1]. This significantly impacts postoperative treatment decisions and provides important prognostic information [2]. In general, lymph node dissection (LND) is a feasible and well-tolerated surgical technique [3].

However, lymphadenectomy is a special procedure that usually faces some difficulties due to many factors as patients' comorbidities, prolonged anesthesia and need of well-trained experienced gynecologic oncologist. For instance, obesity acts as a potential liability to certain surgical procedures. It may adversely affect a comprehensive surgical staging for ovarian cancer owing to exposure difficulties that subsequently leads to inadequate LND as well as increased intraoperative complications. Moreover, those patients experience increased incidence of obesity-related comorbidities during the postoperative course [4]. Thus, compared to non-obese patients, obese females may not undergo LND in spite of the recommendations of LND benefits in surgical staging of ovarian malignancy [5].

Strong evidence suggests that it is necessary to perform adequate surgical staging for ovarian cancer even if there are technical difficulties hindering proper exposure in obese patients [6]. The process of lymphadenectomy in ovarian cancer patients has been associated with greater overall 5-year survival in both early and advanced stage disease [7].

This study discussed the effect of obesity on the number of lymph nodes harvested during systematic LND and the LND-related complications in obese ovarian cancer patients.

\section{Materials and Methods}

This study enrolled women with ovarian cancer who were consecutively subjected to open surgical resection that included systematic LND (pelvic and paraaortic). The exclusion criteria were patients who underwent extra-peritoneal LND or lymph node sampling. The surgeries were performed by oncosurgeons who are specialized in gynecological oncology surgeries at Oncology Center in Mansoura University (OCMU) during the period between January 2012 and June 2017. Data were retrospectively collected from our patients computerized 
registry system including all operative, pathological, radiological and perioperative data.

It was the policy of our cancer center to perform a systematic LND (pelvic and paraaortic) in all patients with ovarian cancer who undergo initial surgery. However, we omitted systematic lymphadenectomy for advanced ovarian cancer cases, restricting it to removing only bulky lymph nodes after the early results of the LION trial (Lymphadenectomy in ovarian neoplasm) were published. Thus, we reviewed only the advanced ovarian cancer cases before our practice shift from systematic lymphadenectomy to removal of bulky nodes [8].

In an attempt to ensure the quality and the standard of the surgical procedures provided to our patients, surgeries were performed by or under the supervision of one of the surgeons in the specialized gynecology oncology team. Thus, all the study group patients were operated, more or less, by the same surgical technique.

All patients underwent exploratory laparotomy with a midline vertical incision extending from the symphysis pubis to just above the umbilicus or the xiphisternum. Lymphadenectomy was done after cytology, completion of total abdominal hysterectomy, bilateral salpingoopherectomy and omentectomy. Systematic LND (pelvic and paraaortic) was performed after exposure of the retroperitoneal space starting caudally from the iliac vessels then heading cranially skeletonizing the inferior vena cava then the aorta and removing all the fibro-fatty tissue with the lymph nodes till skeletonization of the vessels. We used either cold scissors or electrocautery during LND. Bilateral pelvic LND included; external iliac lymphadenectomy followed by exposure of the obturator fossa up to the level of bifurcation of the common iliac vessels. The para-aortic LND (PALND) included; dissection of the lymphatics from the common iliac vessels up to the level of left renal vein crossing the aorta to drain into the inferior vena cava and laterally to the psoas muscle and the ureters.

The LND-related intraoperative complications were identified as vascular, intestinal and bladder injuries. The postoperative complications were identified as wound problems, lymphorrhea or lymphocele formation and thromboembolic events.

Preoperative weights and heights of patients were measured. The BMI was calculated by dividing the weight in kilogram $(\mathrm{kg})$ by the square of the height in meters $(\mathrm{m})$. Patients were categorized according to the recommendations of World Health Organization by their BMI as non-obese $\left(\mathrm{BMI}<30.0 \mathrm{~kg} / \mathrm{m}^{2}\right)$ and obese (BMI $\left.\geq 30.0 \mathrm{~kg} / \mathrm{m}^{2}\right)$ [9].

The number of harvested lymph nodes in the abovementioned stations and in total as well as the LND-related operative complications (intra- and postoperative) were compared amongst the two study groups. The statistical software SPSS version 23 (SPSS Inc., Chicago, IL, USA) was used. For comparing results between the study groups, t-test was used. Complication rates were analyzed using chi-square test or Fisher's exact test. Test results were counted statistically sig- 
nificant when $\mathrm{P}$-value was $<0.05$.

\section{Results}

Seventy-seven women with ovarian cancer were enrolled in the study according to our inclusion and exclusion criteria. The median age of patients was 58 years (IQR 51 - 46 years). The median BMI was $31 \mathrm{~kg} / \mathrm{m}^{2}$ (IQR $28-34 \mathrm{~kg} / \mathrm{m}^{2}$ ). Basic patients' characteristics are demonstrated in Table 1.

Regarding the preoperative BMIs, 43 females (55.8\%) were grouped as obese and $34(44.2 \%)$ as non-obese. Lymph nodes retrieved in total and in different stations separately (pelvic and para-aortic) were all similar among patients in both groups.

LND-related intraoperative complications were observed in 8 patients (18.6\%) in the obese group and 3 patients in the non-obese group $(8.8 \%)(P=0.347)$. They included; vascular injury in 3 patients in the obese group (6.9\%) and 3 patients in the non-obese group (8.8\%), urological injury in 4 patients of the obese group (9.3\%) and bowel injury in one patient in the obese group (2.3\%).

Table 1. Descriptive analysis of the patients enrolled in the study.

\begin{tabular}{|c|c|}
\hline No. of patients & 77 \\
\hline Age, median (IQR) & $58(51-64)$ \\
\hline BMI $\left(\mathrm{Kg} / \mathrm{m}^{2}\right)$, median (IQR) & $31(28-34)$ \\
\hline \multicolumn{2}{|l|}{ Pathology, No. (\%) } \\
\hline Serous & $65(84.5 \%)$ \\
\hline Mucinous & $7(9 \%)$ \\
\hline Brenner & $2(2.5 \%)$ \\
\hline Endometrial & $2(2.5 \%)$ \\
\hline Clear cell & $1(1.5 \%)$ \\
\hline \multicolumn{2}{|l|}{ Grade, No. (\%) } \\
\hline Low grade & $23(30 \%)$ \\
\hline High grade & $39(50.5 \%)$ \\
\hline Not evaluated & $15(19.5 \%)$ \\
\hline \multicolumn{2}{|l|}{ Stage, No. (\%) } \\
\hline I & $28(36.5 \%)$ \\
\hline II & $15(19.5 \%)$ \\
\hline III & $31(40 \%)$ \\
\hline IV & $2(2.5 \%)$ \\
\hline Missing & $1(1.5 \%)$ \\
\hline Patients received neoadjuvant treatment, No. (\%) & $24(31 \%)$ \\
\hline Operative time in minutes, median (IQR) & $195(165-253)$ \\
\hline No. of blood transfusion units, median (IQR) & $1(1-2)$ \\
\hline No. of total LN harvested, median (IQR) & $28(24-31)$ \\
\hline No. of para-aortic LN harvested, median (IQR) & $14(11-18)$ \\
\hline No. of iliac LN harvested, median (IQR) & $14(12-16)$ \\
\hline Postoperative hospital stay in days, median (IQR) & $4(3-5)$ \\
\hline
\end{tabular}


In the entire cohort, LND-related mortalities were not reported. All iatrogenic injuries were controlled by the surgical oncology team with no assistance from other surgeons from other related disciplines except for one case of external iliac artery injury when a vascular surgeon joined for consultation.

Vascular complications ( $\mathrm{n}=6 / 77,7.8 \%$ ) included inferior vena cava injury in 3 cases, a small puncture in the aorta in one case, external iliac vein injury in another and external iliac artery injury in a third case. All vascular injuries occurred during LND and were repaired primarily using 5/0 polypropylene sutures except for the case of external iliac artery injury where a segment of the artery was resected with graft interposition in a conjoint work with a vascular surgeon.

Small Bowel injury occurred in one case in the obese group (2.3\%) during retraction for paraaortic LND which is considered a grade III intraoperative complication by MSKCC (Memorial Sloan Kettering Cancer Center) Surgical Secondary Events (SSE) system. This was full thickness injury of an ileal segment. It was managed with limited primary resection anastomosis. Oral intake in this case was initiated with no signs of intolerance.

Urological complications occurred in 4 patients in the obese group (9.3\%). Urinary bladder injury occurred in 3 cases during retraction for iliac lymphadenectomy which is considered a grade II intraoperative complication by MSKCC (SSE) system as it was repaired immediately in two layers using $3 / 0$ absorbable (polyglactin) sutures and a Silicone catheter was used for 10 days postoperatively to allow completion of the healing process. One case of ureteric injury happened during left iliac LND which is considered a grade III intraoperative complication by MSKCC (SSE) system as it was repaired immediately using 5/0 polyglactin sutures over a stent, then removed by a cystoscope 2 weeks postoperatively. The number of blood units transfused in both groups was the same with a median of one whole blood unit transfused intraoperative (1-2 units in the non-obese group and 0-2 units in the obese group).

The operative time was significantly longer in the obese group with a median of 205 minutes (IQR 170 - 250 minutes) in comparison to 193 minutes in the non-obese group (IQR 148 - 278 minutes).

There was no significant difference in the number of harvested para-aortic and pelvic lymph nodes (LNs) in both groups ( $\mathrm{P}=0.87, \mathrm{P}=0.13$ respectively). The LND had a median count of 14 paraaortic LNs (IQR 11 - 17) and 13 pelvic LNs (IQR 11 - 19) in the obese group, while in the non-obese group 14 paraaortic LNs (IQR 11 - 19) and 15 pelvic LNs (IQR 12 - 16) were harvested.

Postoperative hospital stay was the same in the two groups with a median of 4 days (IQR 3 - 5). Regarding the postoperative LND-related complications, there was statistically significant difference between the two groups Table 2. Postoperative complications occurred in 13 patients $(30.2 \%)$ in the obese group and only in one patient $(3 \%)$ in the non-obese group $(\mathrm{P}=0.004)$. Wound problems occurred in 7 cases in the obese groups compared to one case in the other group. Lymphorrhea occurred only in the obese group $(n=2)$. Similarly, thromboembolic 
Table 2. Comparative analysis between the two groups of the study.

\begin{tabular}{cccc}
\hline & $\begin{array}{c}\text { BMI } \leq 30 \\
(\text { No. }=34)\end{array}$ & $\begin{array}{c}\text { BMI > 30 } \\
(\text { No. }=43)\end{array}$ & P-value \\
\hline Operative time in minutes, median (IQR) & $193(148-278)$ & $205(170-250)$ & 0.45 \\
Total No. of LN harvested median, (IQR) & $28(23-31)$ & $28(26-33)$ & 0.5 \\
No. of harvested para-aortic LN median, & $14(11-17)$ & $14(11-19)$ & 0.87 \\
(IQR) & & & \\
No. of harvested iliac LN median, (IQR) & $13(11-16)$ & $15(12-16)$ & 0.13 \\
No. of blood transfusion units & $1(1-2)$ & $1(0-2)$ & 0.99 \\
Hospital stay (in days) median, (IQR) & $4(3-5)$ & $4(3-5)$ & 0.7 \\
Intraoperative complications n, (\%) & $3(8.8 \%)$ & $8(18.6 \%)$ & 0.347 \\
Vascular complications & $3(8.8 \%)$ & $3(6.9 \%)$ & 0.76 \\
Urological complications & 0 & $4(9.3 \%)$ & 0.58 \\
GIT complications & 0 & $1(2.3 \%)$ & 0.16 \\
Postoperative complications & $1(2.9 \%)$ & $13(30.2 \%)$ & 0.004 \\
Lymphorrhea & 0 & $2(4.6 \%)$ & 0.2 \\
$\quad$ Wound complications & $1(2.9 \%)$ & $7(16.2 \%)$ & 0.057 \\
DVT/Pulmonary embolism & 0 & $4(9.3 \%)$ & 0.068 \\
\hline
\end{tabular}

events were encountered in 4 cases of the obese group and none in the non-obese group.

\section{Discussion}

Obesity is considered nowadays a major health problem with gnisir prevalence all over the world. It is associated with a greater incidence of comorbidities including diabetes mellitus, hypertension, cardiovascular and pulmonary diseases [10]. Even if these comorbidities did not render the patient inoperable, they would probably cause postoperative complications. Additionally, excess abdominal wall fat may cause technical difficulties such as limited field exposure that hinders extensive surgical interventions and can be associated with higher incidence of perioperative complications [4].

Systematic lymph node dissection has been for long considered a corner stone for ovarian cancer surgery for both early and advanced cases. However, after the publication of the LION trial results, it is still considered corner stone for surgical staging in early ovarian cancer patients, whilst removal of bulky lymph nodes in advanced cases as a part of cytoreduction is still to be performed [8].

Lymph node dissection has been shown to increase the survival of ovarian cancer patients [11]. This could be attributed to different factors. Resecting more LNs increases the chance of finding LN metastasis and even micrometastatic disease. Thus, resecting this (clinically undetected) micrometastatic LN disease will definitely affect the decision to receive adjuvant therapies for the clinically early stage ovarian cancer (30\% are upstaged with lymphadenectomy) [12].

In the past, obese patients with ovarian malignancy were less likely to have a systematic lymphadenectomy than non-obese patients [13]. However, further 
studies concluded that the ability to perform adequate LND was not significantly impaired in patients with high BMI (BMI $\geq 30.0 \mathrm{~kg} / \mathrm{m}^{2}$ ) and median lymph node yields were similar irrespective of the BMI category [14]. In our study, we found that the lymph nodes harvested (in every station separately as well as in total) is the same in obese and non-obese patients.

On the contrary, it may be expected that performing lymph node dissection in obese patients with meticulous dissection around the great vessels with a more difficult field exposure will cause more intraoperative and postoperative complications than the same procedure being performed in non-obese patients. Fortunately, comprehensive surgical staging involving systematic lymphadenectomy was found to be performed safely for ovarian cancer obese patients with no statistically significant difference regarding the LND-related intraoperative complication rates [6] between both groups. Vascular injuries were most of these complications followed by urological and bowel injuries. However, no transfusions were required in any of these complications and they were all repaired by the same operating team. Nevertheless, the postoperative complications in the obese group were significantly higher than the non-obese group because these complications are related to the obesity rather than the type of surgery performed.

We believe that there are some potential limitations to this study. This study is a retrospective cohort that involved a relatively small number of patients.

All systematic lymphadenectomies were performed in a single hospital by one team that used the same surgical technique to evade the bias of the potential influence of different approaches of lymph nodes' harvest and its complications. Also, the lymph nodes were examined by the same pathology team. Thus, our work reports that obesity may not be the only factor that determines the safety and adequacy of the operation as regard to the number of lymph node yield, and the perioperative complications in ovarian cancer patients. In addition, major surgical endpoints such as operative time, blood transfusion as well as operative complications were more or less the same in the two groups.

To conclude, obesity per se should not be considered the only factor that pushes the surgeon to omit lymph node dissection or shift to lymph node sampling due to fear of technical difficulties or more complications. The decision to proceed for a systematic lymphadenectomy, sampling or removal of bulky lymph nodes should be solely based on oncological basis. Obese ovarian cancer patients may safely undergo comprehensive staging involving extensive lymph node dissection in open surgeries without significant increase in the rates of intraoperative complications. Whereas, postoperative complications (wound infection, deep venous thrombosis and pulmonary embolism) tend to occur at higher rates with obese patients.

\section{Competing Interests}

The authors declare that they have no competing interests. 


\section{References}

[1] Mikami, M. (2014) Role of Lymphadenectomy for Ovarian Cancer. Journal of Gynecologic Oncology, 25, 279-281. https://doi.org/10.3802/jgo.2014.25.4.279

[2] Gao, J., Yang, X. and Zhang, Y. (2015) Systematic Lymphadenectomy in the Treatment of Epithelial Ovarian Cancer: A Meta-Analysis of Multiple Epidemiology Studies. Japanese Journal of Clinical Oncology, 45, 49-60.

https://doi.org/10.1093/jjco/hyu175

[3] O’Hanlan, K.A., Sten, M.S., O’Holleran, M.S., Ford, N.N., Struck, D.M. and McCutcheon, S.P. (2015) Infrarenal Lymphadenectomy for Gynecological Malignancies: Two Laparoscopic Approaches. Gynecologic Oncology, 139, 330-337. https://doi.org/10.1016/j.ygyno.2015.09.019

[4] Papadia, A., Ragni, N. and Salom, E.M. (2006) The Impact of Obesity on Surgical Outcomes : A Review. International Journal of Gynecological Cancer, 26, 944-952. https://doi.org/10.1111/j.1525-1438.2006.00577.x

[5] Chan, J.K., et al. (2007) The Potential Therapeutic Role of Lymph Node Resection in epithelial Ovarian Cancer: A Study of 13,918 Patients. British Journal of Cancer, 96, 1817-1822. https://doi.org/10.1038/sj.bjc.6603803

[6] Kumar, A., Bakkum-Gamez, J.N., Weaver, A.L., McGree, M.E. and Cliby, W.A. (2014) Impact of Obesity on Surgical and Oncologic Outcomes in Ovarian Cancer. Gynecologic Oncology, 135, 19-24. https://doi.org/10.1016/j.ygyno.2014.07.103

[7] Zhou, J., Shan, G. and Chen, Y. (2016) The Effect of Lymphadenectomy on Survival and Recurrence in Patients with Ovarian Cancer: A Systematic Review and Meta-Analysis. Japanese Journal of Clinical Oncology, 46, 718-726. https://doi.org/10.1093/jjco/hyw068

[8] Harter, P., et al. (2017) LION: Lymphadenectomy in Ovarian Neoplasms-A Prospective Randomized AGO Study Group Led Gynecologic Cancer Intergroup Trial. Journal of Clinical Oncology, 35, 5500.

[9] Calle, E.E. and Kaaks, R. (2004) Overweight, Obesity and Cancer: Epidemiological Evidence and Proposed Mechanisms. Nature Reviews Cancer, 4, 579-591. https://doi.org/10.1038/nrc1408

[10] Upadhyay, J., Farr, O., Perakakis, N., Ghaly, W. and Mantzoros, C. (2018) Obesity as a Disease. Medical Clinics of North America, 102, 13-33. https://doi.org/10.1016/j.mcna.2017.08.004

[11] Aletti, G.D., Dowdy, S., Podratz, K.C. and Cliby, W.A. (2006) Role of Lymphadenectomy in the Management of Grossly Apparent Advanced Stage Epithelial Ovarian Cancer. American Journal of Obstetrics \& Gynecology, 195, 1862-1868. https://doi.org/10.1016/j.ajog.2006.06.068

[12] Kim, H.S., et al. (2010) Systematic Lymphadenectomy for Survival in Epithelial Ovarian Cancer: A Meta-Analysis. International Journal of Gynecological Cancer, 20, 520-528. https://doi.org/10.1111/IGC.0b013e3181d6de1d

[13] Fleury, A., Kushnir, C., Giuntoli, R. and Diaz-Montes, T. (2012) Ovarian Cancer in the Overweight and Obese: Disparities in Cytoreductive Surgery. Gynecologic Oncology, 127, S23. https://doi.org/10.1016/j.ygyno.2012.07.063

[14] Salman, M.C., Usubutun, A., Ozlu, T., Boynukalin, K. and Yuce, K. (2010) Obesity Does Not Affect the Number of Retrieved Lymph Nodes and the Rate of Intraoperative Complications in Gynecologic Cancers. Journal of Gynecologic Oncology, 21, 24-28. https://doi.org/10.3802/jgo.2010.21.1.24 